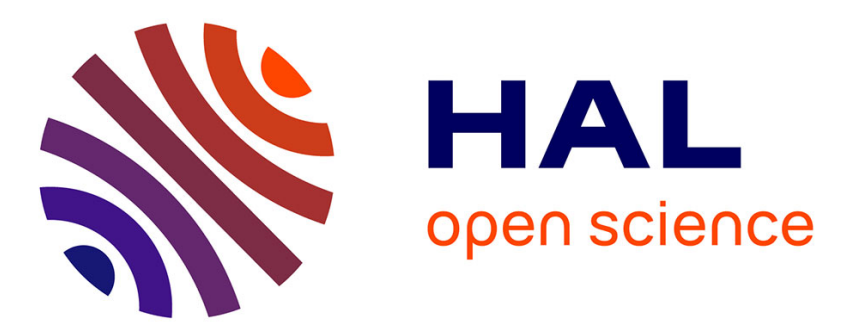

\title{
Improved direction finding using a maneuverable array of directional sensors
}

\author{
Houcem Gazzah, Jean-Pierre Delmas, Sérgio M. Jesus
}

\section{To cite this version:}

Houcem Gazzah, Jean-Pierre Delmas, Sérgio M. Jesus. Improved direction finding using a maneuverable array of directional sensors. ICASSP 2015: 40th International Conference on Acoustics, Speech and Signal Processing, Apr 2015, Brisbane, Australia. pp.2799 - 2803, 10.1109/ICASSP.2015.7178481 . hal-01285357

\section{HAL Id: hal-01285357 \\ https://hal.science/hal-01285357}

Submitted on 9 Mar 2016

HAL is a multi-disciplinary open access archive for the deposit and dissemination of scientific research documents, whether they are published or not. The documents may come from teaching and research institutions in France or abroad, or from public or private research centers.
L'archive ouverte pluridisciplinaire HAL, est destinée au dépôt et à la diffusion de documents scientifiques de niveau recherche, publiés ou non, émanant des établissements d'enseignement et de recherche français ou étrangers, des laboratoires publics ou privés. 


\title{
IMPROVED DIRECTION FINDING USING A MANEUVERABLE ARRAY OF DIRECTIONAL SENSORS
}

\author{
Houcem Gazzah ${ }^{1}$, Jean Pierre Delmas ${ }^{2}$ and Sérgio M. Jesus ${ }^{3}$ \\ ${ }^{1}$ Dept. of Electrical and Computer Engineering, University of Sharjah, UAE, hgazzah@ sharjah.ac.ae \\ ${ }^{2}$ Telecom SudParis, Dept. CITI, CNRS UMR 5157, Evry, France, jean-pierre.delmas@it-sudparis.eu \\ ${ }^{3}$ LarSys, Universidade do Algarve, 8005-139 Faro, Portugal, sjesus@ualg.pt.
}

\begin{abstract}
An antenna array of directional sensors offers more degreesof-freedom to improve the source localization accuracy. The originally sophisticated expression of the CRB turns into a factorized one at high SNR, perfectly suitable to derive an objective function that depends on the array geometry and the source direction PDF. Analytically untractable, optimization is conducted by systematic search for a two-sensor array. The so-optimized array has a finite accuracy in all directions, contrarily to fixed-geometry linear arrays, and a performance comparable to larger-sized fixed-geometry circular arrays, and so at all SNR levels.
\end{abstract}

Index Terms - Cramer Rao bounds, direction-of-arrival estimation, Cardioid sensors

\section{INTRODUCTION}

Accuracy of Direction-of-arrival (DOA) estimation depends on the estimation algorithm, but also on the array geometry. The potential of array geometry adaptation has been recently demonstrated for deterministic [1], random [2, 3], far and near [4] field sources. So far, only arrays of omni-directional sensors have been considered. Arrays of directional sensors pose a number of additional challenges. First, it involves sensor orientations in addition to sensor positions. Second, in the analytical expressions, geometric parameters (sensors positions and orientations) are mixed up with received signal characteristics (notably, signal and noise power). The latter are not to be taken into consideration because they both i) may not be (accurately) known and ii) make the optimization procedure less attractive since less general.

This difficulty is circumvented by considering high observation Signal-to-Noise Ratio (SNR). Surprisingly enough, the subsequently optimized antenna array geometries maintain good performance at arbitrary SNR levels. In order to preserve optimality, and given the analytical complexity of the derived objective function, we proceed by systematic search. At the same time, and in order to reduce the computation burden, only a very limited number of sensors can be accommodated. Interestingly, such short aperture arrays are practical enough to be easily embarked on surveillance platforms such as the increasingly popular and now off-the-shelf autonomous underwater vehicles (AUV) used, e.g., in adaptive sampling networks $[5,6]$.

The widely-accepted Cramer-Rao Bound (CRB) is used as our performance measure to evaluate and optimize the accuracy of the DOA estimate using an array of two directional sensors, with focus on the worst case scenario of no (uniform) source DOA prior. Because we consider the average array performance (at every possible look direction), we use the Expected CRB (ECRB) [7] to build an objective function that involves the array geometry and the Probability Density Function (PDF) of the source DOA [8, 3, 2]. The so-obtained geometry-optimized two-sensor array is compared to the Uniform Circular Array (UCA) of three or more sensors. The UCA is made of the same type of directional sensors, placed regularly along the circle but oriented in such a way that the array itself is isotropic, meaning that the CRB is the same at all look directions. Simulation results show that in typical cases, the proposed two-sensor array exhibits an accuracy comparable to that of UCA of 4 sensors, and so at any SNR level, despite this information not fed to the optimization procedure.

The paper is organized as follows. In Sec. 2, we introduce the observation model and develop expressions of the (high-SNR) CRB. In Sec. 3, the CRB of the array of two directional sensors is studied in details and a subsequent array geometry procedure is defined. The so-optimized array is tested and compared to larger-sized UCAs in Sec. 4. Finally, a conclusion is given in Sec. 5 .

\section{SIGNAL MODEL AND GENERAL RESULTS}

A far-field source is emitting a narrow-band signal $s(t)$ at wavelength $\lambda$ towards an array of $M$ sensors placed in the $[O, x, y)$ plane. Sensor $m$ is placed at a distance $\rho_{m} \lambda$ from the origin $O$ and has a radiation pattern $g_{m}(\theta)$, non-necessarily omni-directional. We let $\phi_{m}$ (resp. $\theta$ ) be the angle (resp. the DOA angle), measured counter-clockwise, that sensor $m$ (resp. the source) forms with the $[O, x)$ axis. 
The vector-valued array output $\mathbf{x}(t)$ collected at time $t$ is given by $\mathbf{x}(t)=\mathbf{a}(\theta) s(t)+\mathbf{n}(t)$, where $\mathbf{a}(\theta)$ is the steering vector whose components are

$$
[\mathbf{a}(\theta)]_{m}=g_{m}(\theta) \exp \left[2 j \pi \rho_{m} \cos \left(\theta-\phi_{m}\right)\right] .
$$

The amplitude of the source $s(t)$ and the noise $\mathbf{n}(t)$ are uncorrelated. $(\mathbf{n}(t))_{t_{1}, . ., t_{N}}$ are independent, zero-mean circular Gaussian distributed with covariance $\sigma_{n}^{2} \mathbf{I} . \quad(s(t))_{t_{1}, \ldots, t_{N}}$ are independent, zero-mean circular Gaussian distributed with variance $\sigma_{s}^{2}$. Under these assumptions, the CRB associated with the observations $(\mathbf{x}(t))_{t_{1}, \ldots, t_{N}}$ is given after concentration on the parameter $\theta$ alone, by $\operatorname{CRB}(\theta)=F^{-1}(\theta)$ with

$$
\frac{F(\theta)}{c}=\left\|\mathbf{a}^{\prime}(\theta)\right\|^{2}\|\mathbf{a}(\theta)\|^{2}-\left|\mathbf{a}^{\prime H}(\theta) \mathbf{a}(\theta)\right|^{2}
$$

with $\mathbf{a}^{\prime}(\theta) \hat{=} d \mathbf{a}(\theta) / d \theta$. Unfortunately, because of the nonfactorized form of

$$
c \hat{=} \frac{2 N \sigma_{s}^{4}}{\sigma_{n}^{2}\left(\sigma_{n}^{2}+\|\mathbf{a}(\theta)\|^{2} \sigma_{s}^{2}\right)},
$$

estimation performance (and any subsequent optimization) will require (and depend on) the knowledge of the source and noise powers. However, if we assume that high SNR is encountered at all possible DOAs, i.e.

$$
\|\mathbf{a}(\theta)\|^{2} \sigma_{s}^{2} \gg \sigma_{n}^{2}
$$

then, we obtain $c \simeq 2 N \sigma_{s}^{2} /\left[\sigma_{n}^{2}\|\mathbf{a}(\theta)\|^{2}\right]$ and

$$
\begin{aligned}
F(\theta) & \simeq \frac{2 N \sigma_{s}^{2}}{\sigma_{n}^{2}}\left(\left\|\mathbf{a}^{\prime}(\theta)\right\|^{2}-\frac{\left|\mathbf{a}^{\prime} H(\theta) \mathbf{a}(\theta)\right|^{2}}{\|\mathbf{a}(\theta)\|^{2}}\right) \\
& \hat{=} \frac{2 N \sigma_{s}^{2}}{\sigma_{n}^{2}} \widetilde{F}(\theta),
\end{aligned}
$$

where $\widetilde{F}(\theta)$ is a convenient design criterion because independent from the noise/signal power.

\section{THEORETICAL DEVELOPMENT}

We propose to optimize the geometry of an array of two directional sensors. One sensor is fixed at the origin. The other one is at distance $\rho \hat{=} \rho_{2}$ and an angle $\phi=\phi_{2}$ which are to be determined by the optimization procedure, along with the sensor orientations. The steering vector being

$$
\mathbf{a}(\theta)=\left[g_{1}(\theta), g_{2}(\theta) \exp [2 j \pi \rho \cos (\theta-\phi)]\right]^{T},
$$

one can prove that

$$
\frac{1}{g_{1}^{4}(\theta)} \frac{F(\theta)}{c}=\left[h^{\prime}(\theta)\right]^{2}+4 \pi^{2} \rho^{2} h^{2}(\theta) \sin ^{2}(\theta-\phi),
$$

where $h(\theta) \hat{=} g_{2}(\theta) / g_{1}(\theta)$. It is worth noticing that the source can be at the array end-fire direction, and yet, the Fisher information can be large if $h^{\prime}(\theta)$ is large. In other words, sources that are in the array end-fire direction can be identified only if we use different and/or differently oriented sensors.

Following similar steps, the high-SNR matrix $F(\theta)$ can be expressed in a similar fashion.

$$
\widetilde{F}(\theta)=g_{1}^{2}(\theta) \frac{\left[h^{\prime}(\theta)\right]^{2}+4 \pi^{2} \rho^{2} h^{2}(\theta) \sin ^{2}(\theta-\phi)}{1+h^{2}(\theta)} .
$$

In practice, we are likely to use identical sensors pointing in different directions, i.e. $g_{m}(\theta) \hat{=} g\left(\theta-\psi_{m}\right)$. Then,

$$
\widetilde{F}(\theta)=\frac{\left[\frac{g^{\prime}\left(\theta-\psi_{1}\right)}{g\left(\theta-\psi_{1}\right)}-\frac{g^{\prime}\left(\theta-\psi_{2}\right)}{g\left(\theta-\psi_{2}\right)}\right]^{2}+4 \pi^{2} \rho^{2} \sin ^{2}(\theta-\phi)}{\frac{1}{g^{2}\left(\theta-\psi_{1}\right)}+\frac{1}{g^{2}\left(\theta-\psi_{2}\right)}}
$$

We consider cardioid-type sensors, of common use in acoustic systems [9], characterized by a directive response of the form $g(\theta)=g_{0}+g_{1} \cos (\theta)$. We update the above expressions in terms of the sensor directivity $\beta \hat{=} g_{1} / g_{0}$, as follows

$$
\begin{aligned}
& {\left[\frac{1}{\left[1+\beta \cos \left(\theta-\psi_{1}\right)\right]^{2}}+\frac{1}{\left[1+\beta \cos \left(\theta-\psi_{2}\right)\right]^{2}}\right] \frac{\widetilde{F}(\theta)}{g_{0}^{2}}} \\
& \quad=\beta^{2}\left[\frac{\sin \left(\theta-\psi_{1}\right)}{1+\beta \cos \left(\theta-\psi_{1}\right)}-\frac{\sin \left(\theta-\psi_{2}\right)}{1+\beta \cos \left(\theta-\psi_{2}\right)}\right]^{2} \\
& \quad+4 \pi^{2} \rho^{2} \sin ^{2}(\theta-\phi)
\end{aligned}
$$

The above tells about the performance of the array at a given source direction. For a source that is randomly located in the array far-field, a suitable measure of the average array performance is the so-called expected $\mathrm{CRB}$ defined as $\mathrm{ECRB} \hat{=} \mathrm{E}(\mathrm{CRB})[8,2,3]$. If the $\operatorname{PDF} f(\theta)$ associated with the source DOA is known, then ECRB $=\int_{0}^{2 \pi} \frac{f(\theta)}{\widetilde{F}(\theta)} d \theta$ is approximately equal to $\frac{\sigma_{n}^{2}}{2 N \sigma_{s}^{2}} \int_{0}^{2 \pi} \frac{f(\theta)}{F(\theta)} d \theta$. We define

$$
\bar{C} \hat{=} \int_{0}^{2 \pi} \frac{f(\theta)}{\widetilde{F}(\theta)} d \theta,
$$

and adopt it as our cost function of the array geometric parameters $\psi_{1}, \psi_{2}, \phi, \rho$. More conveniently, we minimize

$$
g_{0}^{2} \bar{C}=\int_{0}^{2 \pi} \frac{g_{0}^{2}}{\widetilde{F}(\theta)} f(\theta) d \theta
$$

which, thanks to (1), is function of $\beta$. Inter-sensors spacing is assumed to be fixed based on considerations other than estimation accuracy (e.g. coupling and ambiguity considerations). The above cost function is minimized by a 3D systematic search to determine $\operatorname{argmin}_{\phi, \psi_{1}, \psi_{2}} g_{0}^{2} \bar{C}$ characterizing the optimum array. 


\section{NUMERICAL OPTIMIZATION}

The proposed array (optimized based on the high-SNR ECRB) and the reference arrays (UCA of different sizes) are compared in terms of the exact ECRB. Sensors of the UCA, placed at $\phi_{m}=2 \pi(m-1) / M, m=1, \cdots, M$, are to be oriented such that the UCA is isotropic despite being made of directional (cardioid type) sensors. We prove that this is the case if the UCA is made of 5 or more sensors, pointed such that $\phi_{m}=\psi_{m}$, i.e in the direction opposite to the origin. The proposed array will be mentioned as CAM $2^{1}$. In all examples, half-a-wavelength inter-sensors spacing is assumed, in order to avoid (first-order) array ambiguities [10, 11]. Unless otherwise stated, cardioid sensors are considered with $g_{0}=1$ and $g_{1}=0.6$. The optimization problem depends on the particular distribution of the source azimuth angle. We, successively, assume uniform and (mix of) normal distributions to address worst case and realistic scenarios, respectively. The observation SNR is defined as the ratio $\sigma_{s}^{2} / \sigma_{n}^{2}$. Since the two-sensor UCA has an unpractical ECRB (infinite for a uniformly distributed source DOA), our reference, instead, is the three-sensor UCA.

\subsection{Uniform PDF}

In the case of a uniformly distributed source DOA, the problem can be shown to be invariant by rotation of the array, and optimization can be conducted while imposing $\phi=0$. Systematically, the optimal array has its two sensors pointed in opposite directions, orthogonally to the axis linking the two sensors. We denote as CAMU the two-sensor array characterized by $\phi=0, \psi_{1}=\pi / 2$ and $\psi_{2}=3 \pi / 2$, as one optimal array for a source with uniformly distributed DOA.

Contrarily to the two-sensor UCA, the CAMU array does not have an infinite CRB at any direction, as illustrated in Fig. 1. Fig. 2 shows that, as the directivity of the constituent sensors increases, the CRB is reduced in the end-fire direction and is increased at broadside. In fact, the CAMU array best emulates an isotropic array when the sensors directivity is close to 0.5, as confirmed in Fig. 3. Overall, in terms of the $\mathrm{ECRB}$, performance of the CAMU array is finite, comparable to that of the larger three-sensor UCA, as illustrated by Fig. 3 . As can be concluded from Fig. 3, the use of directive sensors is more beneficial to CAMU than to UCA3 array.

Clearly, optimally-configured two-sensor array is outperformed by the larger three-sensor UCA. This is true because, disadvantageously, this PDF expresses no prior about the source DOA. However, as shown in the next section, the situation is different if (more) information is available about the source DOA.

\footnotetext{
${ }^{1}$ We compare this two-sensor array to the chameleon whose eyes can rotate and move independently from each other.
}

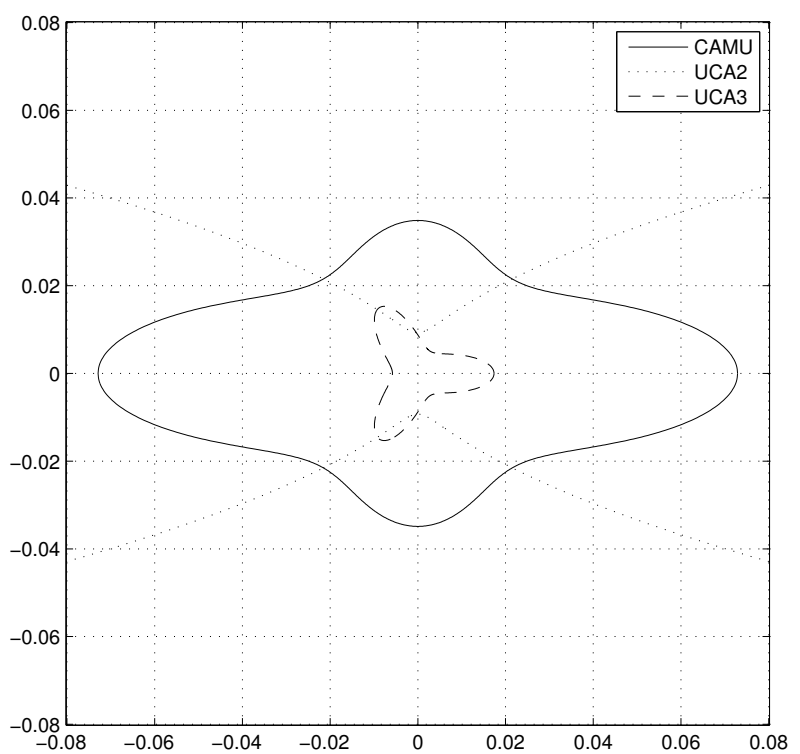

Fig. 1. Polar representation of $N \times C R B$, for all possible source DOAs and at an SNR of $10 \mathrm{~dB}$.

\subsection{Normal PDF}

In realistic scenarios, $\mathrm{PDF}$ is modeled as a mixture of $P$ Gaussian distributions with different means (standing for the different look directions) and variances (expressing uncertainties about the look directions). Let $\kappa_{p}, \Omega_{p}$ and $\sigma_{p}$ be the weight, the mean and the standard deviation of the $p$-th distribution, so that

$$
f(\theta)=\sum_{p=1}^{P} \frac{\kappa_{p}}{\sigma_{p} \sqrt{2 \pi}} \exp \left[-\frac{\left(\theta-\Omega_{p}\right)^{2}}{2 \sigma_{p}^{2}}\right],
$$

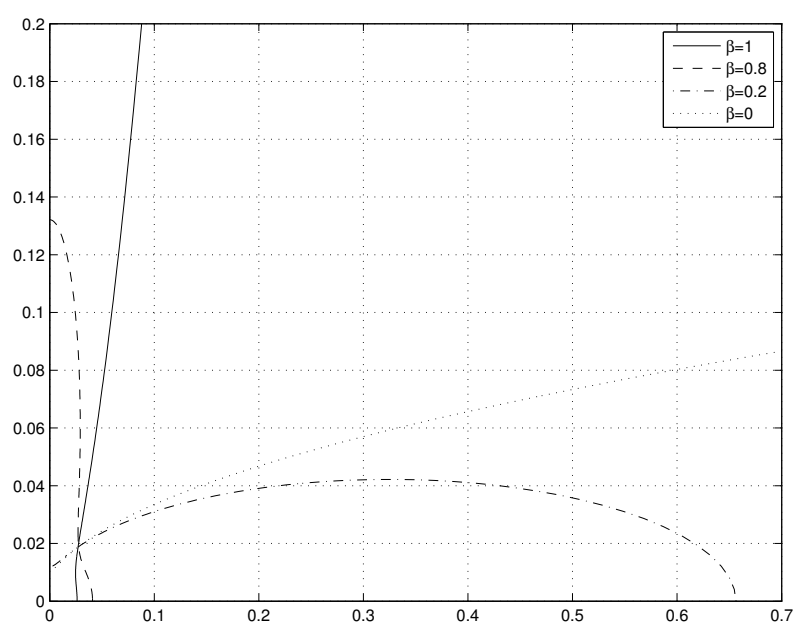

Fig. 2. Impact of the sensors directivity on the CAMU array for which $N \times C R B$ is shown in polar reprentation, for all possible source DOAs and an SNR of $10 \mathrm{~dB}$. 


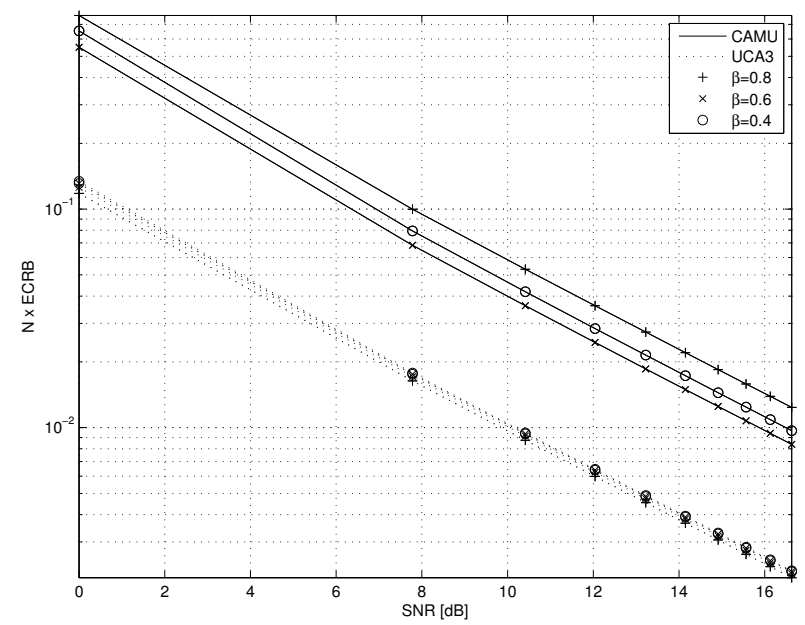

Fig. 3. Impact of the sensors directivity on the ECRB for a uniformly distributed source DOA.

where $\sum_{p=1}^{P} \kappa_{p}=1$. We choose $\sigma_{p} \ll 2 \pi$ and/or $0 \ll \Omega_{p} \ll$ $2 \pi$ in order to have $f(\theta) \simeq 0$ for any $\theta$ not in $[0,2 \pi]$. In the simulations, we have assumed equally likely look directions, i.e. $\kappa_{1}=\cdots=\kappa_{P}=1 / P$ and the same uncertainty, i.e. $\sigma_{1}=\cdots=\sigma_{P}=5[$ DEG $]$.

First, for a particular source PDF defined by $\Omega_{1}=281$, $\Omega_{2}=310$ and $\Omega_{3}=61$ [DEG], the best two-sensor array is found to be the one characterized by $\phi=3.6, \psi_{1}=345.6$ and $\psi_{2}=349.2$ [DEG], achieving a performance close to that of the 4-sensor UCA, at any SNR level, as clear from Fig. 4. It is worth noticing that the CAMU array performs well, despite not being meant for this particular PDF.

Second, in a set of simulations, and in order to explore the performance of the proposed array for arbitrary PDFs, we assume two possible look directions: $\Omega_{1}=\pi$ and $\Omega_{2}$ rang-

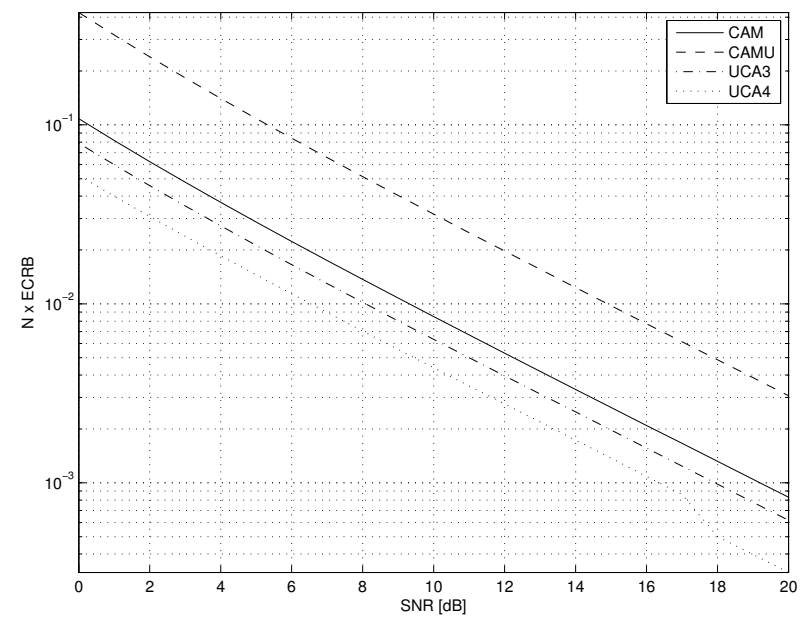

Fig. 4. Compared performance of the optimized CAM array and the reference UCAs, for a particular distribution of the source DOA.

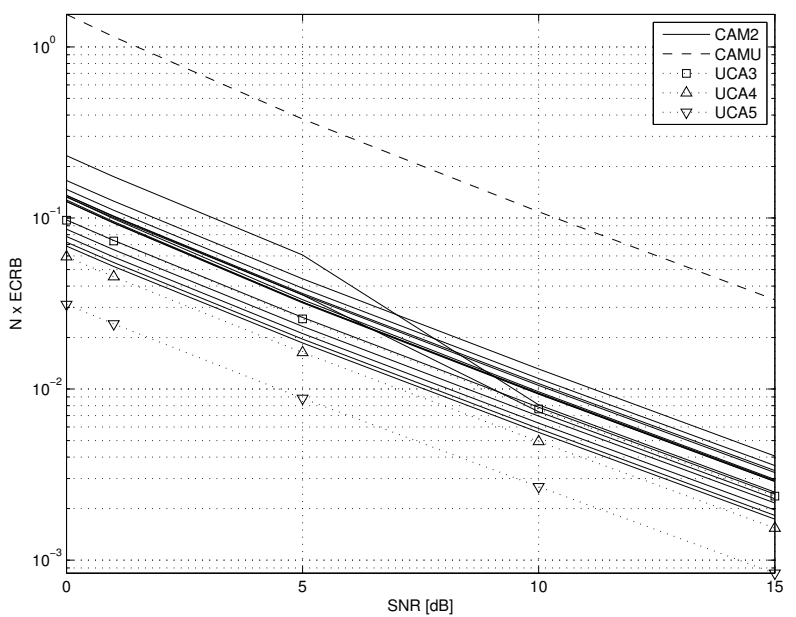

Fig. 5. Compared performance of CAM, CAMU, and UCA arrays for a source PDF given by $\Omega_{1}=0$ and $\Omega_{2}$ spanning $20,40, \ldots, 160$ [DEG]. Cardioid sensors are used for which $\beta=0.4$.

ing from 20 to 160 [DEG]. As illustrated in Fig. 5, we compare the optimized arrays (in solid lines) to reference UCAs (in doted lines). We realize that, overall, the optimized twosensors array performs closely to the 3 -sensor UCA, regardless of the observation SNR. Performance of the CAMU array continues to be comparable to those of its competitors, despite that this array does not take benefit from the available information (PDF) about the source DOA.

\section{CONCLUSION}

We use two directive sensors and set their positions and pointing directions according to the statistical information available about the target DOA. By doing so, we compensate for a major drawback of linear arrays, that of being unable to detect sources at the end-fire direction, rendering linear arrays as useful as planar arrays. It is shown via simulations that the proposed algorithm outperforms the generic uniform circular array with a larger number of sensors. This minimally-sized array could be, for instance, suitable for deployment as part of autonomous underwater vehicles or to determine the optimal geometry of a swarm of vehicles for use in tasks such as environmental monitoring and geophysical exploration. 


\section{REFERENCES}

[1] H. Gazzah and K. Abed-Meraim, "Optimum Ambiguityfree directional and omni-directional planar antenna arrays for DOA estimation," IEEE Trans. Signal Processing, vol. 57, no. 10, pp. 3942-3253, Oct. 2009.

[2] O. Lange and B. Yang, "Optimization of array geometry for direction-of-arrival estimation using a priori information," Adv. Radio Sci., vol. 8, pp. 87-94, 2010.

[3] H. Gazzah and J. P. Delmas, "Direction finding antenna arrays for the randomly located source," IEEE Trans. Signal Process., vol. 60, no. 11, pp. 6063-6068, Nov. 2012.

[4] H. Gazzah and J. P. Delmas, "CRB based-design of linear antenna arrays for near-field source localization," IEEE Trans. Antennas Propag., vol. 62, no. 4, pp. 1965-1974, April 2014.

[5] D. Wang, P. F.J. Lermusiaux, P. J. Haley, D. Eickstedt, W. G. Leslie, H. Schmidt, "Acoustically focused adaptive sampling and on-board routing for marine rapid environmental assessment," Elsevier Journal of Marine Systems, 78 (2009), pp. S393âS407.

[6] H. Schmidt, J. G. Bellingham and J. W. Bales, "Mobile underwater arrays," U.S. Patents No. US Patent 5,894,450 (April 1999). https://encrypted.google.com/patents/US5894450.

[7] H. L. Van Trees and K. L. Bell, Bayesian Bounds for Parameter Estimation and Nonlinear Filtering/Tracking, $\mathrm{H}$. L. Van Trees and K. L. Bell, Ed. John Wiley \& Sons, 2007.

[8] Ü. Oktel and R. L. Moses, "A Bayesian approach to array geometry design," IEEE Trans. Signal Process., vol. 53, no. 5, pp. 1919â1923, May 2005.

[9] L. del Val, A. Izquierdo, M. I. Jimenez, J. J. Villacorta and M. Raboso, "Analysis of Directive Sensor Influence on Array Beampatterns," in "Microwave and Millimeter Wave Technologies Modern UWB antennas and equipment," I. Mini (Ed.), InTech. Available: http://www.intechopen.com/books/microwave-andmillimeter-wave-technologies-modern-uwb-antennasandequipment/analysis-of-directive-sensor-influence-onarray-beampatterns.

[10] L. C. Godara and A. Cantoni, "Uniqueness and linear independence of steering vectors in array space," J. Acoust. Soc. Amer., vol. 70, no. 2, pp. 467-475, Aug. 1981.

[11] M. Gavish and A. J. Weiss, "Array geometry for ambiguity resolution in direction finding," IEEE Trans. Antennas and Propag., vol. 44, no. 6, pp. 143-146, Feb. 1991. 